\title{
Association between virtues and posttraumatic growth: Preliminary evidence from a Chinese community sample after earthquakes
}

Wenjie Duan, Pengfei Guo

Objective: Relationship, vitality, and conscientiousness are three fundamental virtues that have been identified recently, which are important individual differences to health, well being, and positive development. This cross-sectional study attempted to explore the relationship between the three constructs and post-traumatic growth (PTG) in three directions, including indirect trauma samples without post-traumatic stress disorder (PTSD), direct trauma samples without PTSD, and direct trauma samples with PTSD. Methods: A total of 340 community participants from Sichuan Province, Mainland China involved in the study, most of which experienced Wenchuan and Lushan Earthquake. Participants were required to complete the self-reported questionnaire packages at one time point for obtaining their scores on virtues (Chinese Virtues Questionnaire), PTSD (PTSD Checklist-Specific), and PTG (Post-traumatic Growth Inventory-Chinese). Results: Significant and positive correlations between the three virtues and PTG were identified $(r=$ .39 to $.56 ; p<.01)$. Further regression analysis by stepwise method reveled that: in the indirect trauma samples, vitality explained $32 \%$ variance of PTG. In reference to the direct trauma sample without PTSD, both relationship and conscientiousness explained $32 \%$ variance of PTG; whereas in the direct trauma sample with PTSD, only conscientiousness accounted for $31 \%$ the variance in PTG. Conclusion: This cross-sectional investigation partly revealed the roles of different virtues in trauma context. Findings suggest important implications for strengths-based treatment. 
11 Address for correspondence: Pengfei Guo, Hospital (T. C. M.) Affiliated to Luzhou Medical College,

12 Luzhou, Sichuan, China. (Telephone: +86-152-9829-9409; Email: lyzyydb@163.com) 
13 Association between Virtues and Posttraumatic Growth: Preliminary Evidence from a Chinese

14

15

16

17

18

19

20

21

22

23

24

25 31 hope, and religiousness show stronger relations with PTG than other strengths (Peterson et al. 2008). 32 However, no correlation coefficient was higher than 0.35 , thus reflecting weak correlation. Principal

\section{Community Sample after Earthquakes}

\section{INTRODUCTION}

Natural disasters, cancer, bereavement, and other life-threatening events with potentially

irreversible consequences often lead to positive changes and transcendence known as posttraumatic growth (PTG, Calhoun \& Tedeschi 2006; Tedeschi \& Calhoun 2004). A promising research direction focuses on the effects of personal strengths on coping and responses to health- and wellbeing-related concerns and worries, such as traumatic events (Hampson \& Friedman 2008).

To date, the most systematic approach for studying personal strengths is the Values in Action

Classification developed by Peterson \& Seligman (2004); this approach included 24 character strengths

(e.g., hope, self-regulation, and gratitude) grouped into six core virtues (e.g., wisdom, courage, and humanity). They conceptualized virtue as "a property of the whole person and the life that person leads" (p. 87), which is a personal strength appreciated by the whole society (Peterson \& Seligman 2004). In the past decade, many studies revealed the positive relationship between these positive qualities and mental health (for review, see Niemiec 2013) and demonstrated that the use of strengths is a valid approach for enhancing wellbeing in diverse populations (Duan et al. 2014; Seligman et al. 2005). Accordingly, the relationship between these qualities and PTG was explored using 1,739 samples from different countries (Peterson et al. 2008). Findings indicated that kindness, love, bravery, 
33 component factor analysis revealed a five-factor structure of virtues, namely, interpersonal, fortitude,

34 cognitive, transcendence, and temperance. Correlation analysis showed that all these five virtues

35 correlated with PTG, but all the correlation coefficients were lower than 0.21 (Peterson et al. 2008).

36 Three issues should be noted regarding the study of Peterson et al. (2008). First, the virtue

37 structure in the aforementioned study is questionable. Various studies have found that the 24 identified

38 strengths can be grouped into different virtues in diverse cultures, and the groups include 3-, 4-, or 5-

39 factor structures (Duan et al. 2012b; Ho et al. 2014a). Thus, the virtue structure should be clarified

40 prior to delineating the function of virtues in facing trauma and should be explored and analyzed

41 further. Duan et al. (2012b) adopted the combined emic and etic approaches to select 96 cross-

42 culturally equivalent items from the original 240-item Values In Action Inventory of Strengths by

43 using a Chinese population (Ho et al. 2014b). Exploratory and confirmatory factor analyses revealed 3

44 virtues, namely, relationship, vitality, and conscientiousness (Duan et al. 2013; Zhang et al. 2014a).

45 The relationship virtue reflects "the love, concern, and gratitude of a person toward others"; vitality

46 reflects "the curiosity and zest for creativity of an individual"; and conscientiousness is "an

47 intrapersonal virtue that describes people who persist in achieving goals and exhibit self-control,"

48 which reflects the individual orientation of the virtues (Ho et al. 2014a). A recent study conducted by

49 McGrath (in press) likewise demonstrated the three-virtue structure by investigating multiple

50 inventories and large samples. Therefore, the relationship between the three virtues and PTG should be

51 reexamined. Second, the research conducted by Peterson et al. (2008) examined only the relationship

52 among trauma samples, which accounted for $56 \%$ of the entire population. However, previous studies 
53 argued that stress-related life events could likewise facilitate stress-related growth, which is assessed

54 by Posttraumatic Growth Inventory (PTGI), but to a lesser extent than traumatic events (e.g., LoSavio

55 et al. 2011). The level of perceived stress that resulted from events might be the key cause of PTG,

56 rather than the objective and specific events per se. Third, several individuals who underwent trauma

57 may develop posttraumatic stress disorder (PTSD), whereas others may not (Yehuda \& Flory 2007).

58 The above study failed to consider the influence of PTSD on PTG in the trauma samples. An inverted-

59 U curve was also found between PTG and PTSD, which suggested that PTG decreased after a

60 moderate level of PTSD (Levine et al. 2008). Nevertheless, an increasing interest was noted among

61 mental health professionals in determining the strengths of their clients (McCrae 2011).

62

\section{CURRENT STUDY}

63 To expand our understanding of the function of strengths in PTG, the relationship between

64 virtues and PTG should be explored. Basing on the above literature review, PTG in the current study

65 refers to growth following stress-related events, including daily stressors and traumatic events.

66 Individuals indirectly exposed to traumatic events can be recognized as persons who experienced

67 stress-related events. Accordingly, both direct and indirect trauma groups would acquire PTG, and the

68 differences between these two groups would be insignificant (Hypothesis 1). As previously discussed,

69 a few traumatic individuals may develop PTSD, which implied that the contributions of the three

70 virtues (relationship, vitality, and conscientiousness) to PTG vary depending on trauma type (i.e.,

71 direct trauma vs. indirect trauma) and PTSD status (i.e., PTSD group vs. non-PTSD group) 
72 (Hypothesis 2). The current results will clarify the contributions of virtues in traumatic situations,

73 which may facilitate a strength-based approach in both research and practice in the future.

\section{METHOD}

75 Participants

76

A total of 340 qualified respondents (109 males and 231 females) were recruited from different

77 communities in Dujiangyan area, Sichuan, China, which were affected by the 2008 earthquake. A total

78 of 69 participants were aged $18-25,101$ were in the range of $36-35,112$ were in the range of $36-45$,

79 and 58 were above 46 years old. Only 51 participants have obtained a university degree or above. As

80 expected, "natural disaster," "sudden or unexpected death of someone close to you," and "life-

81 threatening illness or injury" were the three most endorsed items listed on the questionnaire on 82 traumatic events (Table 1).

83 (Insert Table 1 Here)

\section{Procedures} The announcement for study participation was published on community bulletin boards, which

86 can be seen by most people who lived in the community. Individuals who were interested to participate

87 were instructed to complete first the Life Events Checklist (LEC), and only the participants who

88 directly or indirectly experienced trauma were qualified to complete the other scale during the

89 following week (screening criteria are described in the Measures section). The Human Subjects

90 Committee of Traditional Chinese Medicine Hospital Affiliated to Luzhou Medical College approved 
91 the study. All data collected were anonymous and confidential. Psychological assistance was provided

92 to protect the subjects. Data were collected from December 2013 to April 2014.

93 Measures

Life Events Checklist. LEC was used to screen individuals who experienced direct or indirect

95 trauma through 17 potential events (Gray et al. 2004). Participants are requested to rate each event on a

96 five-point Likert scale $(1=$ happened to me, $2=$ witnessed it, $3=$ learned about it, $4=$ not sure, $5=$

97 does not apply). Participants who indicated at least one traumatic event as $1=$ "happened to me" were

98 defined as direct trauma samples, whereas respondents who indicated $2=$ "witnessed it" and/or $3=$

99 "learned about it" were indirect trauma samples. Participants who selected 4 = "not sure" and/or 5

100 "does not apply" in the checklist were excluded. Considering that all participants involved in this study

101 were sampled within the earthquake zone (Dujiangyan area in Sichuan Province), rather than other

102 place far away from the earthquake-prone area, they should not be treated as persons who lived far

103 away from Sichuan and were affected by the earthquake only through radio and television. Thus, all

104 the qualified participants may have experienced at least some indirect exposure to earthquake.

105 Chinese Virtues Questionnaire. Virtues were assessed using the Chinese Virtues Questionnaire,

106 which is a 96-item simplified Chinese scale with good psychometric characteristics (Duan et al. 2013;

107 Duan et al. 2012b). The respondents were requested to rate each item from 1 (very much unlike me) to

1085 (very much like me) on a five-point Likert scale. Item samples include "I can accept love from

109 others" (Relationship), "I like to think of new ways to do things" (Vitality), and "I control my

110 emotions" (Conscientiousness). A high mean score reflects a high degree of the virtue within an 
111 individual. In this study, the Cronbach's $\alpha$ values of the three subscales were 0.91 (relationship), 0.85

112 (vitality), and 0.84 (conscientiousness).

113 Posttraumatic Growth Inventory-Chinese. A 15-item Chinese version of the PTGI (Ho et al.

114 2004) measures growth following a traumatic event. The measurement requires individuals to indicate

115 the extent of their experiences of changes as a result of crisis, ranging from 0 (not at all) to 5 (a very

116 great degree). The reliability and validity of the 15 -item version were accurate in previous studies (Ho

117 et al. 2004). In the present sample, the Cronbach's $\alpha$ of the inventory was 0.84 .

$118 \quad \boldsymbol{P C L}-\boldsymbol{S}$. PTSD symptoms were evaluated by the 17-item PCL-S. Participants are requested to

119 rate their experience from 1 (not at all) to 5 (extremely). Previous studies demonstrated that the

120 Chinese version can be used as a screening questionnaire among the Chinese population ( $\mathrm{Li}$ et al.

121 2010). Scores of 44 or above indicate a PTSD diagnosis (Blanchard et al. 1996). Responses of the

122 diagnosed PTSD participants also fulfilled the criterions of DSM-IV, including a) "history of a

123 traumatic stressor," b) "persistent re-experiencing of the traumatic event," c) "persistent avoidance of

124 stimuli associated with the trauma and numbing of general responsiveness," and d) "persistent

125 symptoms of increased arousal." In the current sample, the Cronbach's $\alpha$ of the entire scale was 0.93 .

\section{RESULTS}

The descriptive statistics of all variables are listed in Table 2. ANOVA showed that the virtue

128 of relationship and PTG exhibited significant differences among the three samples $(p<0.05)$. Post-hoc

129 tests further revealed that both the relationship and PTG of direct trauma with PTSD sample were

130 significantly lower than those of the other groups $(p<0.05)$. Correlation analysis (Table 3 ) revealed 
131 that different virtues showed significantly positive relations with PTG in the total sample and three 132 subsamples, ranging from 0.39 to $0.56(p<0.01)$.

138 PTSD sample, relationship (Beta $=0.38, t=5.98, p<0.001)$ and conscientiousness $($ Beta $=0.29, t=$

$1394.54, p<0.001)$ also accounted for $32 \%$ variance of PTG. Finally, in the direct trauma with PTSD

140 sample, only conscientiousness (Beta $=0.56, t=3.85, p<0.01$ ) can contribute $31 \%$ explained variance 141 to PTG.

\section{DISCUSSION}

145 trauma (e.g., earthquakes). As expected, no difference of PTG existed between the indirect and direct experienced an earthquake, whereas some might have indirectly experienced this event through their

149 friends, witnessed the death of a close friend, or experienced the psychological distress caused by the 150 death of a family member or a close friend. Thus, all the participants may undergo PTG. The growth 
151 also decreased with increased severity of PTSD, which was partly explained by the inverted-U curve

152 between PTG and PTSD. As reflected by the present results, the correlation between PTG and PTSD

153 was almost significant and negative, particularly in the PTSD sample $(r=-0.31, p=0.07)$. Mol et al.

154 (2005) also investigated 832 individuals and found that the scores of their PTSD were the same for

155 some early-life and traumatic events, which partly revealed that some life events could also generate

156 PTSD symptoms. All these results suggest that the objective traumatic events may not essentially lead

157 to PTG or PTSD. According to the Transactional Model of Stress and Coping (Lazarus \& Folkman

158 1984), the perceived stress from these events could be triggered. Thus, further studies are necessary to

159 understand the differences in PTG and PTSD after traumatic and non-traumatic events.

160 Our previous studies preliminarily demonstrated that three virtues are positively associated with

161 positive health outcomes (Duan et al. 2012a; Duan et al. 2013; Tang et al. in press), such as satisfaction

162 with life and flourishing, but negatively associated with negative health outcomes (Duan et al. 2013;

163 Duan et al. 2015; Zhang et al. 2014c), such as depression, anxiety, general severity index, and

164 pathological Internet use. These results indicated that the three virtues might be protective factors of

165 mental health. Accordingly, personal virtues may facilitate rebounding for individuals who experienced

166 trauma.

Individuals with different endorsed virtues often occupy different psychological resources (e.g.,

168 optimism, emotional control, and gratitude). Thus, they can maximize the use of different resources

169 (i.e., diverse virtues) in various contexts. For instance, an individual with high level of relationship

170 virtue is more adept at obtaining social support from his/her friends and relatives to recover from the 
171 trauma and even obtain growth. Thus, the main results of the current study revealed the different

172 potential functions of virtues (i.e., relationship, vitality, and conscientiousness) in PTG. In the indirect

173 trauma samples, only vitality contributed to the variance of growth after trauma. In the direct trauma

174 without PTSD sample, relationship and conscientiousness explained the variance of PTG, whereas in

175 the PTSD sample, only conscientiousness was the significant contributor. Basing on the previous and

176 current findings, we can speculate the different functions of virtues in various samples. Without

177 directly experiencing trauma, most people are troubled by small stress, and some are indirectly affected

178 by trauma. When similar scenarios occur, vitality can cause individuals to perceive less stress, thereby

179 reducing psychological distress (Duan et al. 2015). Our previous study identified that only students

180 with higher vitality have perceived less stress from minor life events, which consequently introduced

181 less psychological distress (Duan et al. 2015). Individuals with high vitality are also more willing to

182 express their concerns to relieve stress and improve mental health (Yang et al. 2015; Zhang et al.

183 2014b). After being directly exposed to traumatic events, most people retain psychological balance

184 without significant symptoms of PTSD (Bonanno et al. 2007). Therefore, interpersonal resources

185 related to the virtue of relationship are necessary. Prati \& Pietrantoni (2009) demonstrated that social

186 support is a significant contributor to PTG in a meta-analysis of 103 studies. Individuals who are rated

187 high in the virtue of relationship are more likely to adopt a supportive mechanism to overcome the

188 predicaments caused by trauma. Finally, conscientiousness, also termed as self-regulation, always

189 facilitates positive mental health and decreases psychopathology (Hagger 2010). Duan et al. (2015)

190 found that individual conscientiousness can directly decrease psychological distress, regardless of the 
191 level of stress. Consequently, the intrapersonal strengths reflected by conscientiousness can be used to 192 regulate emotion, cognition, and behavior to resolve conflicts caused by trauma, thus enhancing growth 193 after trauma.

194 In fact, the three virtues (i.e., relationship, vitality, and conscientiousness) researched here 195 correspond strongly to the three well-established character traits proposed by Cloninger \& Zohar 196 (2011), namely cooperativeness, which is consistent with relationship; self-directedness, which is 197 consistent with vitality; and self-transcendence, which is consistent with conscientiousness. These 198 character traits and the three virtues, according to the Cloninger (2004)'s psychobiological theory of 199 personality, were cognitive domain of personalities, which were more stable and developed across 200 lifespan toward social norms that approved and appreciated by the whole society (Josefsson et al. 201 2013b). Previous studies found that high self-directedness as a health-promoting trait that associated 202 with coping with prior trauma (North et al. 2012). Other longitudinal studies revealed the predictive 203 ability of character traits to well being (Cloninger \& Zohar 2011; Josefsson et al. 2013a). These 204 findings and abovementioned studies on virtues suggest that both character traits and virtues may be 205 helpful in coping with challenges and enhancing well-being. For instance, Eley et al. (2013) recently 206 revealed the associations between profile of temperament \& character and resilience, indicating the 207 importance of high levels of self-directedness, cooperativeness, and persistence. Our ongoing cross208 sectional study likewise preliminarily found that virtues and trait resilience were conceptually related 209 (Duan \& Guo under review). However, future longitudinal studies should be operated to explore the 
210 predictive ability of virtues in traumatic context and further reveal the possible causal relationship

211 between virtues and resilience.

212 Some limitations should be noted. First, it should be stressed again that, similar to some studies

213 on trauma, the design of this study was cross-sectional rather than longitudinal. Results of this research

214 and the hypothesized functions of virtues in the development process of a traumatic event should be

215 replicated and examined in future longitudinal designs. Second, the direct and indirect trauma samples

216 were divided based on self-reported measurements, wherein "indirect trauma" could be anything from

217 hearing about the traumatic event from a friend to watching it on TV, although they lived in the

218 earthquake zone. These participants were actually not trauma-exposure types according to DSM or

219 ICD, thereby leaving future studies to distinguish the two samples objectively. Third, all the data were

220 collected by self-reported method, which may introduce common method bias. Future studies should

221 adopt multiple methods for controlling the bias. Finally, this study is the first to examine the function

222 of virtues in trauma research among Chinese. More psychological outcomes, including flourishing,

223 depression, and anxiety, should be explored in the future. 
227 Blanchard EB, Jones-Alexander J, Buckley TC, and Forneris CA. 1996. Psychometric properties of the PTSD Checklist (PCL). Behav Res Ther 34:669-673.

229 disaster? The role of demographics, resources, and life stress. Journal of consulting and clinical psychology 75:671-682.

Calhoun LG, and Tedeschi RG. 2006. Handbook of posttraumatic growth: Research \& practice. Mahwah, NJ: Erlbaum.

Cloninger CR. 2004. Feeling good: the science of well being. New York: Oxford University Press.

Cloninger CR, and Zohar AH. 2011. Personality and the perception of health and happiness. Journal of affective disorders 128:24-32.

Duan W, Bai Y, Tang X, Siu PY, Chan RKH, and Ho SMY. 2012a. Virtues and positive mental health. Hong Kong Journal of Mental Health 38:24-31.

Duan W, and Guo P. under review. Relationships among Trait Resilience, Virtues, Post-traumatic Stress Disorder, and Post-traumatic Growth. PLoS One.

Duan W, Ho SMY, Bai Y, and Tang X. 2013. Psychometric evaluation of the Chinese Virtues Questionnaire. Research on Social Work Practice 23:336-345.

Duan W, Ho SMY, Bai Y, Tang X, Zhang Y, Li T, and Yuen T. 2012b. Factor structure of the Chinese Virtues Questionnaire. Research on Social Work Practice 22:680-688. 
245 Duan W, Ho SMY, Siu BPY, Li T, and Zhang Y. 2015. Role of Virtues and Perceived Life Stress in Affecting Psychological Symptoms among Chinese College Students. Journal of American College Health 63:32-39.

Duan W, Ho SMY, Tang X, Li T, and Zhang Y. 2014. Character strength-based intervention to promote satisfaction with life in the Chinese university context. Journal of Happiness Studies

251 Eley DS, Cloninger CR, Walters L, Laurence C, Synnott R, and Wilkinson D. 2013. The relationship 252 253 between resilience and personality traits in doctors: implications for enhancing well being. PeerJ 1:e216.

254 Gray MJ, Litz BT, Hsu JL, and Lombardo TW. 2004. Psychometric properties of the life events 255 checklist. Assessment 11:330-341. Health Psychology Review 4:57-65.

258 Hampson SE, and Friedman HS. 2008. Personality and health: A lifespan perspective. In: John PO, Robins WR, and Pervin AL, eds. Handbook of personality: Theory and research. 3rd ed. New York, NY, US: Guilford Press.

261 Ho SMY, Chan CLW, and Ho RTH. 2004. Posttraumatic growth in Chinese cancer survivors. 262 Psycho-Oncology 13:377-389. 
263 Ho SMY, Duan W, and Tang SCM. 2014a. The psychology of virtue and happiness in western and asian thought. In: Snow NE, and Trivigno FV, eds. The Philosophy and Psychology of Character and Happiness. New York: Routledge, 215-238.

266 Ho SMY, Rochelle TLR, Law LSC, Duan W, Bai Y, Shih S-M, and Wang G-L. 2014b. Methodological issues in positive psychology research with diverse populations: Exploring strengths among Chinese adults. In: Pedrotti JT, and Edwards LM, eds. Perspectives on the Intersection of Multiculturalism \& Positive Psychology. New York, N.Y.: Springer Science + Business Media B.V., 45-57.

Josefsson K, Jokela M, Cloninger CR, Hintsanen M, Salo J, Hintsa T, Pulkki-Råback L, and Keltikangas-Järvinen L. 2013a. Maturity and change in personality: developmental trends of temperament and character in adulthood. Development and psychopathology 25:713-727.

Lazarus RS, and Folkman S. 1984. Stress, appraisal, and coping. New York: Springer Publishing offspring's temperament and character traits after 18 years. Psychiatry research 209:643-651.

279 Levine SZ, Laufer A, Hamama-Raz Y, Stein E, and Solomon Z. 2008. Posttraumatic growth in adolescence: Examining its components and relationship with PTSD. Journal of Traumatic Stress 21:492-496. 
282 Li H, Wang L, Shi Z, Zhang Y, Wu K, and Liu P. 2010. Diagnostic utility of the PTSD Checklist in detecting PTSD in Chinese Earthquake victims. Psychological Reports 107:733-739. 785.

McCrae RR. 2011. Personality traits and the potential of positive psychology. In: Sheldon KM, forward. New York: Oxford University Press, 193-206.

McGrath RE. in press. Integrating Psychological and Cultural Perspectives on Virtue: The Hierarchical Structure of Character Strengths. Journal of Positive Psychology.

Mol SS, Arntz A, Metsemakers JF, Dinant G-J, AP VILTERS-VAN MONTFORT P, and Knottnerus from an open population study. The British Journal of Psychiatry 186:494-499. directly exposed survivors of the Oklahoma City bombing. Comprehensive psychiatry 53:1-8.

300 Peterson C, Park N, Pole N, D'Andrea W, and Seligman ME. 2008. Strengths of character and posttraumatic growth. Journal of Traumatic Stress 21:214-217. 
302

303

304

305

306

307

308

309

310

311

312

313

Peterson C, and Seligman MEP. 2004. Character strengths and virtues: A handbook and classification:

$$
\text { Oxford University Press, USA. }
$$

Prati G, and Pietrantoni L. 2009. Optimism, social support, and coping strategies as factors contributing to posttraumatic growth: A meta-analysis. Journal of Loss and Trauma 14:364388.

Seligman MEP, Steen TA, Park N, and Peterson C. 2005. Positive psychology progress: empirical validation of interventions. American Psychologist 60:410-421.

Tang X, Duan W, Wang Z, and Liu T. in press. Psychometric Evaluation of the Simplified Chinese Version of Flourishing Scale. Research on Social Work Practice.

Tedeschi RG, and Calhoun LG. 2004. Posttraumatic growth: Conceptual foundations and empirical evidence. Psychological Inquiry 15:1-18.

Yang Z, Tang X, Duan W, and Zhang Y. 2015. Expressive writing promotes self-reported physical, social and psychological health among Chinese undergraduates. International Journal of Psychology 50:128-134.

Yehuda R, and Flory JD. 2007. Differentiating biological correlates of risk, PTSD, and resilience following trauma exposure. Journal of Traumatic Stress 20:435-447.

Yu X, Lau JT, Zhang J, Mak WW, Choi KC, Lui WW, Zhang J, and Chan EY. 2010. Posttraumatic growth and reduced suicidal ideation among adolescents at month 1 after the Sichuan Earthquake. Journal of affective disorders 123:327-331. 
321 Zhang Y, Duan W, Tang X, Gan P, Gan F, and Guo P. 2014a. Psychometric Properties of Chinese Virtues Questionnaire in Chinese Adolescents. Chinese Journal of Clinical Psychology 22:470474.

324 Zhang Y, Duan W, Tang X, and Yang Z. 2014b. Can virtues enhance the benefits of expressive writing among healthy Chinese? A pilot study. Journal of Mental Health 23:231-235.

326 Zhang Y, Yang Z, Duan W, Tang X, Gan F, Wang F, Wang J, Guo P, and Wang Y. 2014c. A preliminary investigation on the relationship between virtues and pathological internet use among Chinese adolescents. Child and Adolescent Psychiatry and Mental Health 8:8. 


\section{Table 1 (on next page)}

Demographic and Sample Characteristics

Table 1 Demographic and Sample Characteristics 
2 Table 1

3 Demographic and Sample Characteristics

\begin{tabular}{|c|c|c|}
\hline Variables & $n$ & $\%$ \\
\hline \multicolumn{3}{|l|}{ Gender } \\
\hline Male & 109 & $32.06 \%$ \\
\hline Female & 231 & $67.94 \%$ \\
\hline \multicolumn{3}{|l|}{ Age } \\
\hline $18-25$ & 69 & $20.29 \%$ \\
\hline $26-35$ & 101 & $29.71 \%$ \\
\hline $36-45$ & 112 & $32.94 \%$ \\
\hline 46 and above & 58 & $17.06 \%$ \\
\hline \multicolumn{3}{|l|}{ Education } \\
\hline Secondary school and below & 37 & $10.88 \%$ \\
\hline Tertiary school & 186 & $54.71 \%$ \\
\hline College & 66 & $19.41 \%$ \\
\hline University and above & 51 & $15.00 \%$ \\
\hline \multicolumn{3}{|l|}{ Types of trauma } \\
\hline Natural disaster & 126 & $37.06 \%$ \\
\hline Sudden, unexpected death of someone close to you & 102 & $30.00 \%$ \\
\hline
\end{tabular}


Life threatening illness or injury

Fire or explosion

Transportation accident
$56 \quad 16.47 \%$

$49 \quad 14.41 \%$

$33 \quad 9.71 \%$ 
Table 2 (on next page)

Descriptive Statistics and Group Differences

Table 2 Descriptive Statistics and Group Differences 
2 Table 2

3 Descriptive Statistics and Group Differences

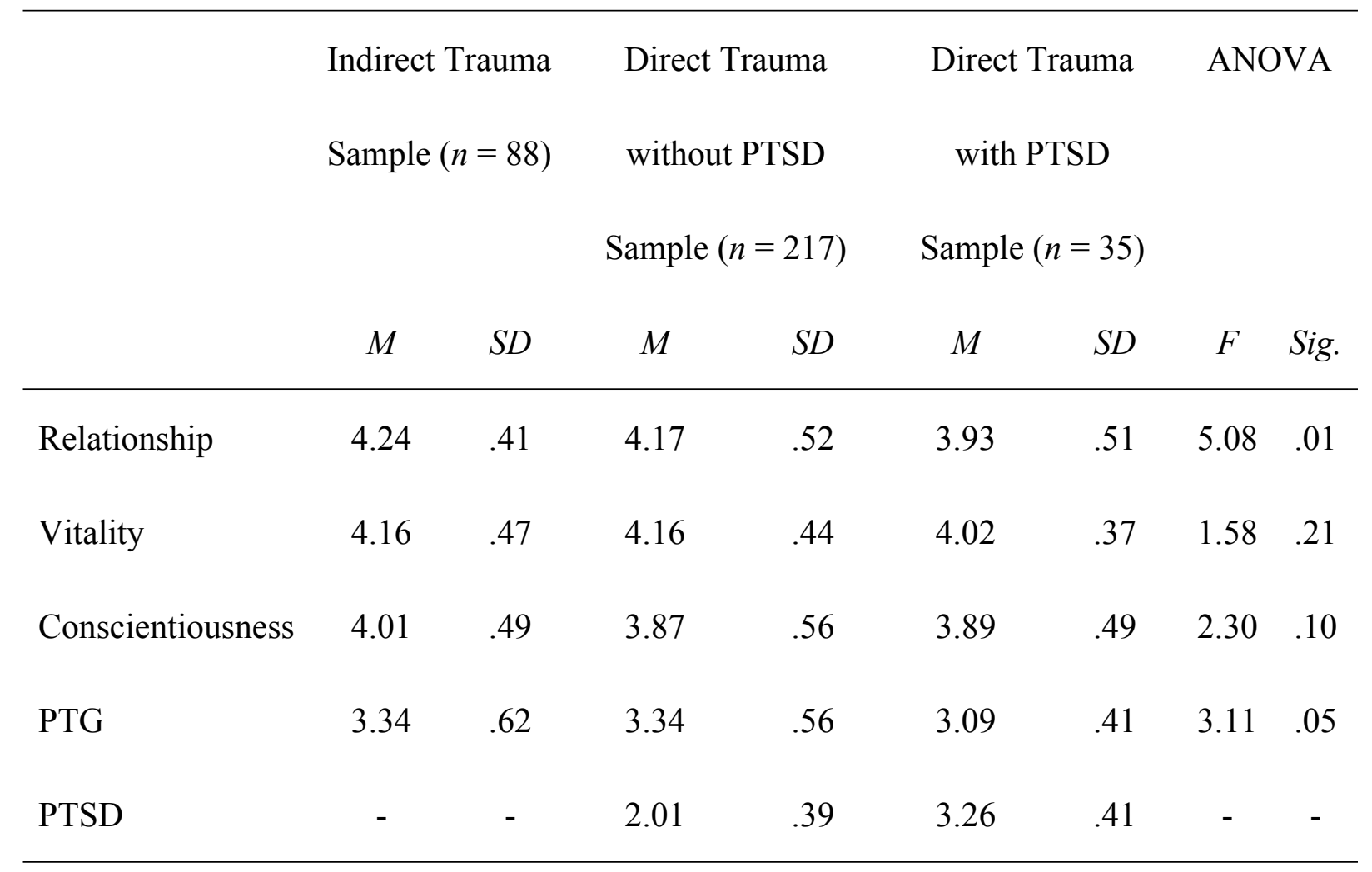

4

5

6 
Table 3 (on next page)

Correlations between Virtues and Posttraumatic Growth in Different Subgroups

Table 3 Correlations between Virtues and Posttraumatic Growth in Different Subgroups 
2 Table 3

3 Correlations between Virtues and Posttraumatic Growth in Different Subgroups

Posttraumatic Growth

Total Indirect Trauma Direct Trauma Direct Trauma

Sample Sample $(n=88) \quad$ without PTSD with PTSD Sample

\begin{tabular}{lcccc} 
& & Sample $(n=217)$ & $(n=35)$ \\
\hline Relationship & $.48^{* *}$ & $.44 * *$ & $.50 * *$ & $.53^{* *}$ \\
Vitality & $.54 * *$ & $.56^{* *}$ & $.39 * *$ & $.45^{* *}$ \\
Conscientiousness & $.46^{* *}$ & $.49 * *$ & $.45^{* *}$ & $.56^{* *}$ \\
\hline
\end{tabular}

$4 \quad * * p<.01$.

5 
Table 4 (on next page)

Regression of Virtues on Posttraumatic Growth in Different Subgroups

Table 4 Regression of Virtues on Posttraumatic Growth in Different Subgroups 
2 Table 4

3 Regression of Virtues on Posttraumatic Growth in Different Subgroups

\begin{tabular}{|c|c|c|c|c|c|c|c|c|c|c|c|c|}
\hline & \multicolumn{4}{|c|}{ Indirect Trauma Sample } & \multicolumn{4}{|c|}{ Direct Trauma without PTSD Sample } & \multicolumn{4}{|c|}{ Direct Trauma with PTSD Sample } \\
\hline & \multicolumn{4}{|c|}{$(n=88)$} & \multicolumn{4}{|c|}{$(n=217)$} & \multicolumn{4}{|c|}{$(n=35)$} \\
\hline & $R^{2}$ & $F$ & Beta & $t$ & $R^{2}$ & $F$ & Beta & $t$ & $R^{2}$ & $F$ & Beta & $t$ \\
\hline & .32 & $40.05 * * *$ & & & .32 & $50.22 * * *$ & & & .31 & $14.827 * *$ & & \\
\hline Relationship & & & .07 & .53 & & & .38 & $5.98^{* * *}$ & & & .27 & 1.29 \\
\hline Vitality & & & .56 & $6.44 * * *$ & & & .07 & 1.04 & & & .23 & 1.36 \\
\hline Conscientiousness & & & .17 & 1.37 & & & .29 & $4.54 * * *$ & & & .56 & $3.85^{* *}$ \\
\hline
\end{tabular}

$4 \quad * * p<.01 ; * * * p<.001$

5 